\title{
Demand functions and demand manifolds
}

\section{Alan Beggs ${ }^{1}$}

Received: 4 March 2021 / Accepted: 26 March 2021 / Published online: 12 April 2021

(c) The Author(s) 2021

\begin{abstract}
Mrázová and Neary (2017) introduce the notion of the Demand Manifold which expresses the relationship between the elasticity and curvature of a demand function. They argue that this determines many important comparative static properties of firm behavior. This paper gives necessary and sufficient conditions for two demand functions to have the same demand manifold and so to have similar comparative static properties.
\end{abstract}

Keywords Demand manifold · Demand functions · Comparative statics

JEL classification C02 - D21

\section{Introduction}

In an interesting recent paper, Mrázová and Neary (2017) argue that many important comparative static properties of firm behavior depend on the relationship between the elasticity of demand and the curvature of demand. They introduce the notion of the 'demand manifold' which summarizes this relationship. Two demand functions with the same demand manifold will have similar predictions for firm behavior, so it is important to know when this is the case. Mrázová and Neary (2017) give a partial characterization of when two demand functions have the same demand manifold. This paper shows that a combination of two conditions they give is, under mild conditions, both necessary and sufficient for two demand functions to have the same demand manifold and so similar comparative static predictions.

The pricing decisions for a firm are determined by the elasticity of demand. How it responds to shocks will depend on the curvature of demand. The relationship between the elasticity and curvature of demand is therefore pivotal in determining comparative statics. Mrázová and Neary (2017) introduce the idea of the demand manifold to

\footnotetext{
Alan Beggs

alan.beggs@economics.ox.ac.uk

1 Department of Economics and Wadham College, Oxford University, Oxford OX1 3PN, UK
} 
summarize this relationship. They argue that this idea sheds light on many important comparative static questions, for example the degree of pass through in monopoly and the effects of globalization on output, prices and welfare in models of monopolistic competition.

Given the importance of the relationship between the elasticity and curvature of demand for comparative static predictions, it is of interest to know when two demand curves have the same demand manifold and so have similar comparative static predictions. This paper shows that two demand curves have the same demand manifold if and only if one can be derived from the other by a change in market size and a change in quality. Changes in market size and in quality will therefore lead to unchanged comparative statics. Changes in demand for other reasons will, however, result in demand curves with different demand manifolds and so potentially different comparative static predictions.

These results help to understand what kinds of changes to the environment may affect comparative statics. Changes in quality and market size will leave the relationship between elasticity and curvature unchanged and so comparative statics will be unaffected. If these are the only kind of changes likely then comparative statics conclusions are robust. Other changes in the environment will affect this relationship and so require more detailed investigation in order to determine whether and in what in way comparative static predictions may be changed.

The importance of the relationship between elasticity and the curvature of demand has been realized in a number of contexts, for example pass-through under monopoly (see for example Bulow and Pfleiderer (1983); Weyl and Fabinger (2013)), price discrimination (see for example Aguirre et al. (2010)) and monopolistic competition (see for example Zhelobodko et al. (2012)). The current paper builds on Mrázová and Neary (2017) which perhaps brings out the issue in the clearest way.

The paper proceeds as follows. Section 2 outlines the results and gives examples of their application to a number of demand systems. Section 3 gives a formal statement of the results. It is shown that, under mild conditions, two inverse demand curves have the same demand manifold if and only if one can be derived from the other by a change in market size and a change in quality. Section 3 also presents the proof of the results and discusses the reasons for the results. Section 4 concludes.

\section{Outline of results and examples}

Consider a monopolist with inverse demand function $P(x)$. It will choose to operate at the point where marginal revenue equals marginal cost or equivalently

$$
P\left(1-\frac{1}{\epsilon}\right)=\mathrm{MC}
$$

where $\epsilon$ is the elasticity of demand. How price changes in response to a small change in costs depends on the elasticity of demand and how this changes as $x$ changes, that is on the curvature of demand. Understanding the relationship between the elasticity and curvature of demand is therefore pivotal for comparative statics. 
Mrázová and Neary (2017) consider an inverse demand function $P(x)$ and look at its elasticity $\epsilon(x)$ and a measure of curvature $\rho(x)$ :

$$
\epsilon(x)=-\frac{P(x)}{x P^{\prime}(x)} \quad \rho(x)=-\frac{x P^{\prime \prime}(x)}{P^{\prime}(x)}
$$

They argue that many important comparative static properties under monopoly or monopolistic competition are determined by the relationship between $\epsilon$ and $\rho$. They consider the set

$$
\mathcal{D}(P)=\{(\epsilon(x), \rho(x))): x \in \operatorname{dom} P\}
$$

which they refer to as the Demand Manifold. dom $P$ is the set $\{x: x>0, P(x)>0\}$. $P$ is decreasing so $\operatorname{dom}(P)$ is an open interval $\left(0, x_{\max }\right)$, where $x_{\max }$ may be finite or infinite.

They ask under what conditions the Demand Manifold is invariant to changes in parameters. Equivalently one can ask, when do two inverse demand functions $P(x)$ and $P^{*}(x)$ have the same demand manifold. Below it will be shown that, under some mild conditions, it must be the case that

$$
P^{*}(x)=a P(b x) \text { for all } x
$$

where $a>0$ and $b>0$ are constants.

Equivalently the demand manifold is independent of a parameter $\theta$ if and, under mild conditions, only if the inverse demand function can be written is

$$
P(x ; \theta)=a(\theta) \bar{P}(b(\theta) x)
$$

Mrázová and Neary (2017) note that if either inverse demand has the form

$$
P(x ; \theta)=a(\theta) \bar{P}(x)
$$

so that demand changes because of changes in quality (in the sense that the prices consumers are willing to pay change by the same factor for all quantities) or the direct demand function has the form

$$
D(p ; \theta)=c(\theta) \bar{D}(p)
$$

so that demand changes because of changes in market size (in the sense that sales change by the same factor for any price), then the demand manifold is independent of $\theta$.

Since the inverse demand function is the inverse of the direct demand function, (5) is simply the combination of (6) and (7). Sufficiency is then clear from Mrázová and Neary (2017). That (4) implies that $P^{*}$ and $P$ have the same demand manifolds can also be easily checked by direct calculation of $\epsilon$ and $\rho$. Multiplication of $P$ by $a$ leaves $\epsilon$ and $\rho$ unchanged while multiplying $x$ by $b$ replaces them by their values at $b x$. In 
either case the relationship between them is unchanged. The novelty in the current paper is that this condition is also, under mild conditions, necessary for invariance of the demand manifold.

This paper works in terms of the inverse demand function. Relabeling variables one can of course obtain exactly analogous results for the direct demand function.

To proceed it is slightly more convenient to work in terms of (minus) one over the elasticity and a different measure of curvature:

$$
\eta(x)=-\frac{1}{\epsilon(x)}=\frac{\partial \ln P}{\partial \ln x} \quad \kappa(x)=\frac{\partial^{2} \ln P}{\partial(\ln x)^{2}}
$$

and the set

$$
\mathcal{E}(P)=\{(\eta(x), \kappa(x)): x \in \operatorname{dom} P\}
$$

$\epsilon$ and $\eta$ are are one-one-correspondence and given these so are $\rho$ and $\kappa$ (see Appendix and the online Appendix of Mrázová and Neary (2017)), so $\mathcal{D}(P)=\mathcal{D}\left(P^{*}\right)$ if and only if $\mathcal{E}(P)=\mathcal{E}\left(P^{*}\right) . \kappa$ is (minus) Kimball (1995)'s superelasticity of demand.

To illustrate the results consider the following examples:

Example 1 Constant Elasticity Demand: $P(x)=A x^{-\delta}$

Here $\mathcal{D}(P)=(1 / \delta, 1+\delta)$ and $\mathcal{E}(P)=(-\delta, 0)$. That is both $\mathcal{D}(P)$ and $\mathcal{E}(P)$ are one-point sets and independent of $A$. Any two inverse demand curves of the CES form with the same value of $\delta$ are multiples of each other, so (4) is satisfied.

Example 2 Bipolar demand: $P(x)=A x^{-\alpha}+B x^{-\beta}$.

This class was introduced by Mrázová and Neary (2017) and encompasses a number of commonly demand systems, for example linear demand $(\alpha=0$ and $\beta=-1)$ and the demand system introduced by Bulow and Pfleiderer (1983) $(\alpha=0, \beta>0)$.

$\alpha=\beta$ reduces to the constant elasticity case, so without loss of generality assume that $\alpha>\beta$. To be a valid demand system, $P$ non-negative and decreasing, one needs (see Appendix) $A>0$ and either $\alpha>0$ or $\alpha=0$ and $B<0$. In addition if $B>0$ one needs $\beta \geq 0$. Assume that $B \neq 0$, otherwise this is again the constant elasticity case.

Some calculation (see Appendix) shows that all points of $\mathcal{D}(P)$ satisfy the equation $\rho=\alpha+\beta+1-\alpha \beta \epsilon$, whereas the points of $\mathcal{E}(P)$ satisfy the equation $\kappa=-(\eta+$ $\alpha)(\eta+\beta)$. Both equations are clearly independent of $A$ and $B$.

The only dependence on $A$ and $B$ is that the sign of $B$ determines the range of $\eta$ (and $\epsilon$ ). If $B>0$ then (see Appendix) $\eta$ takes values between $-\alpha$ and $-\beta$, so $\mathcal{D}(P)=\{(\epsilon, \rho): \rho=\alpha+\beta+1-\alpha \beta \epsilon, 1 / \alpha<\epsilon<1 / \beta\}$ and $\mathcal{E}(P)=\{(\eta, \kappa):$ $\kappa=-(\eta+\alpha)(\eta+\beta),-\alpha<\eta<-\beta$ \}. If $B \leq 0$ (see Appendix) then $\eta$ takes values between $-\infty$ and $-\alpha$ (and $\epsilon$ between 0 and $1 / \alpha$ ). In either case $\mathcal{D}(P)$ and $\mathcal{E}(P)$ are described by the same equations but with the ranges of $\epsilon$ and $\eta$ replaced by these. ${ }^{1}$

\footnotetext{
1 Mrázová and Neary (2017) refer to the two different cases for the sign of $B$ as determining which branch of the demand manifold is relevant, thinking of the entire set described by the equation as the demand manifold. A given inverse demand function produces one branch or the other, however, so here the corresponding branch is treated as the demand manifold for that function.
} 
Condition (4) holds for any pair of the inverse demand curves with the same values of $\alpha$ and $\beta$ provided $B$ and $B^{\prime}$ have the same sign: if

$$
P(x)=A x^{-\alpha}+B x^{-\beta} \quad \text { and } \quad P^{\prime}(x)=A^{\prime} x^{-\alpha}+B^{\prime} x^{-\beta}
$$

then

$$
P^{\prime}(x)=a P(b x)
$$

with

$$
a=\left(A / A^{\prime}\right)^{\frac{\beta}{\alpha-\beta}}\left(B^{\prime} / B\right)^{\frac{\alpha}{\alpha-\beta}}, \quad b=\left(A / A^{\prime}\right)^{\frac{1}{\alpha-\beta}}\left(B^{\prime} / B\right)^{\frac{1}{\alpha-\beta}}
$$

$a$ and $b$ are both positive real numbers if and only if $B$ and $B^{\prime}$ have the same sign since $A$ and $A^{\prime}$ are both positive by assumption. In other words, two demand curves with the same values of $\alpha$ and $\beta$ will have the same demand manifolds provided $B$ and $B^{\prime}$ have the same sign, consistent with the explicit expressions for these above.

Example 3 Inverse exponential demand: $P(x)=A+B \exp \left(-C x^{\delta}\right)$.

Here $B>0, C>0$ and $\delta>0$. Mrázová and Neary (2017) (Online Appendix B.10) show that the demand manifold, $\mathcal{D}(P)$, is given by the equation $\epsilon=\frac{1+\frac{A}{B} \exp \left(\frac{\rho+\delta-1}{\delta}\right)}{\rho+\delta-1}$. In the Appendix it is shown that $\mathcal{E}(P)$ is given by the implicit equation $\eta=$ $\frac{\left(\eta^{2}-\delta \eta+\kappa\right) / \eta}{\frac{A}{B} \exp \left(-\frac{\eta^{2}-\delta \eta+\kappa}{\delta \eta}\right)+1}$. Both these sets are independent of $C$ and depend only on the ratio of $A$ to $B$ not their individual values.

The results here imply that $P^{*}(x)=a P(b x)$ has the same demand manifold (and the same set $\mathcal{E}(P))$ as $P$. Applying this to the form of $P$ implies that the demand manifold depends only on the ratio of $A$ to $B$ (invariance to $a$ ) rather than their individual values and is independent of $C$ (invariance to $b$ ), which was seen by explicit calculation in the previous paragraph.

\section{Results}

This section gives a formal statement of the results and outlines the proof. The idea of the proof is that if two inverse demand functions have the same demand manifold then they have the same relationship between elasticity and curvature or equivalently between (logarithmic) first and second derivatives of (the log of) the inverse demand functions. Given this relationship one can determine the inverse demand curve up to some constants and so two inverse demand curves with the same manifold must be related as in (4). This section makes this idea precise.

It will be assumed that

Assumption 1 For any inverse demand function $P$ considered, $P$ is thrice continuously differentiable on its domain and if for any $x, \kappa(x)=0$ then $\frac{\partial^{3} \ln P}{\partial(\ln x)^{3}}$ is non-zero at that point. 
Under these assumptions the mapping $f(x)=(\eta(x), \kappa(x))$ is a regular curve (see do Carmo (1976)). $\mathcal{E}(P)$ is its image (or trace).

Assumption 2 In addition to Assumption 1, the mapping $f: x \rightarrow(\eta(x), \kappa(x))$ is one-to-one on the domain of $P$.

Under this additional assumption $\mathcal{E}(P)$ is an (immersed) submanifold of $\mathcal{R}^{2} .^{2}$ In particular, the demand manifold does not cross itself.

Assumption 1 is violated by Example 1 and the demand manifold is a point not a curve. The result holds for the constant elasticity example, so Assumption 1 is not necessary, but it is a degenerate case.

Both assumptions are satisfied by Example 2: $\kappa$ never vanishes, so Assumption 1 holds and also $\eta$ is as a consequence monotonic so Assumption 2 holds (see Appendix). For a similar reason both assumptions will hold in general if $\kappa(x)<0$ for all $x$, which is implied by but does not imply $P(x)$ being log-concave, or $\kappa(x)>0$ for all $x$. Mrázová and Neary (2019) label the former case (strict) sub-convexity and the latter super-convexity. Example 3 need not be sub- or super-convex globally but is it shown in the Appendix that Assumptions 1 and 2 are satisfied.

If the domain of the inverse demand function is a finite interval and the inverse demand function is well-defined at the endpoints of the interval, then these are the only assumptions needed. If not, however, a further assumption is needed to guarantee that the demand manifold does not bend back to cross itself in the limit. Let $L_{x \rightarrow 0}(f)$ denote the set of limit points of $f$ as $x \rightarrow 0$ and $L_{x \rightarrow x_{\max }}(f)$ the set of limit points as $f$ tends to the upper endpoint of the domain (which may infinite). If $f$ has well-defined limits as it approaches the endpoints of the domain these sets reduce to these limits but this is not assumed.

Assumption 3 In addition to Assumptions 1 and $2, L_{x \rightarrow 0}(f) \cap \mathcal{E}(P)=\varnothing$ and $L_{x \rightarrow x_{\max }}(f) \cap \mathcal{E}(P)=\varnothing$

Note that $L_{x \rightarrow 0}(f) \cap L_{x \rightarrow x_{\max }}(f) \neq \emptyset$ is allowed.

Under this assumption, $\mathcal{E}(P)$ is a submanifold of $\mathcal{R}^{2}$ in a slightly stronger sense. In particular it rules out the manifold curving back on itself to form a figure eight, say, though only intersecting itself in the limit. Between them Assumptions 2 and 3 guarantee that only one part of the manifold passes through a given point and points close to it. $^{3}$

Assumption 3 is certainly satisfied if the inverse demand function is sub- or superconvex as then $\eta$ is monotonic. In particular it is satisfied in the Bipower case. In the inverse exponential case inverse demand need not be sub- or super-convex but it is shown in the Appendix that Assumption 3 is satisfied.

\footnotetext{
2 An immersed submanifold is the image of a one-one immersion. Under Assumption 1 the derivative of the mapping $f$ never vanishes and so $f$ is an immersion and under Assumption $2 f$ is injective. See Lee (2013) Chapter 5 for discussion of different definitions of submanifolds.

3 More formally under this additional assumption $\mathcal{E}(P)$ is an embedded submanifold of $\mathcal{R}^{2}$. This follows from Lee (2013) Chapter 5 Proposition 5.21 as under this assumption $x \rightarrow(\eta(x), \kappa(x))$ is a proper mapping (the inverse image of a compact set is compact).
} 
Proposition 1 If $P$ and $P^{*}$ both satisfy Assumptions 1 to 3 then $\mathcal{E}(P)=\mathcal{E}\left(P^{*}\right)$, or equivalently $\mathcal{D}(P)=\mathcal{D}\left(P^{*}\right)$, if and only if $P^{*}(x)=a P(b x)$ for all $x \in$ dom $P^{*}$ for some $a>0$ and $b>0$.

To prove the result it is convenient to change variables to $\Pi=\ln P$ and $y=\ln x$. Setting

$$
\mu(y)=\frac{\partial \Pi}{\partial y} \quad v(x)=\frac{\partial^{2} \Pi}{\partial y^{2}}
$$

and

$$
\mathcal{F}(\Pi)=\{(\mu(y), v(y)): y \in \overline{\operatorname{dom}} \Pi\}
$$

where $\overline{\mathrm{dom}} \Pi=\{y: y>-\infty, \Pi>-\infty\}$. That is $\overline{\mathrm{dom}} \Pi$ is an open interval $\left(-\infty, y_{\max }\right)$, where $y_{\max }$ may be finite or infinite. Clearly $\mathcal{F}(\Pi)=\mathcal{E}(P)$.

To prove the result above it is therefore enough to prove that $\mathcal{F}(\Pi)=\mathcal{F}\left(\Pi^{*}\right)$ if and only if

$$
\Pi^{*}(y)=\alpha+\Pi(y+\beta) \text { for all } y
$$

where $\alpha$ and $\beta$ are constants. That is the curve $\Pi^{*}$ is a translation of the curve $\Pi$.

Assumptions 1 to 3 become

Assumption $4 \Pi$ is thrice continuously differentiable on its domain and if for any $y$, $v(y)=0$ then $\frac{\partial^{3} \Pi}{\partial y^{3}}$ is non-zero at that point.

Assumption 5 In addition to Assumption 4, the mapping $g: y \rightarrow(\mu(x), v(x))$ is one-to-one on the domain of $\Pi$.

Assumption 6 In addition to Assumptions 4 and $5, L_{y \rightarrow-\infty}(g) \cap \mathcal{F}(\Pi)=\varnothing$ and $L_{y \rightarrow y_{\max }}(g) \cap \mathcal{F}(\Pi)=\emptyset$

Proposition 2 If $\Pi$ and $\Pi^{*}$ both satisfy Assumptions 1 to 3 then $\mathcal{F}(\Pi)=\mathcal{F}\left(\Pi^{*}\right)$ if and only if $\Pi^{*}(y)=\alpha+\Pi(\beta+y)$ for all $y \in \overline{\text { dom }} \Pi^{*}$ for some $\alpha$ and $\beta$.

Proposition 1 is an immediate corollary of this result.

The idea of the proof is straightforward. Suppose $S=\mathcal{F}(\Pi)=\mathcal{F}\left(\Pi^{*}\right)$ can be written in the form

$$
v=h(\mu)
$$

That is $v$ can be written as a function of $\mu$ along the manifold. Then $\Pi$ and $\Pi^{*}$ satisfy the same second-order differential equation

$$
\Pi^{\prime \prime}=h\left(\Pi^{\prime}\right)
$$


where primes denote derivatives. $\Pi$ and $\Pi^{*}$ may differ in starting point (hence the constant $\beta$ ) and value at the starting point (hence the constant $\alpha$ ) but once these are specified they are uniquely determined by this differential equation.

It may not be possible to write $v$ as a function of $\mu$ globally so the argument in the Appendix is more detailed but this is the essential idea. $v$ can be expressed as a function of $\mu$ locally at points where $\frac{\partial^{3} \Pi}{\partial y^{3}} \neq 0$ and the proofs shows that the resulting local relations between $\Pi$ and $\Pi^{*}$ can be patched together globally.

More geometrically the result can be understood as saying that if two curves have the same relationship between curvature and slope then they must be translations of one another.

The result is related to the Fundamental Theorem of Planes Curves: two plane curves parameterized by arc length have the same curvature if and only if they differ by a translation and a rotation (see do Carmo (1976)). The notion of curvature of a curve there is the standard one in differential geometry. ${ }^{4}$ The curves here are not parameterized by arc length and it is easier to give a direct argument. Proposition 2 does not allow for rotations unlike the Fundamental Theorem of plane curves. This is because in an economic context the axes have intrinsic meaning and rotating the inverse demand curve affects the elasticity of demand, for example, and so economic behavior would be affected even if from a geometric point of view the curves are equivalent.

Assumptions 2 and 3 or equivalently Assumptions 5 and 6 can probably be relaxed but some restriction is necessary. Consider the following example:

\section{Example 4}

$$
\Pi(y)=2 \pi a-a y+\sin (y) \quad y \in[0,2 \pi]
$$

and

$$
\Pi^{*}(y)=4 \pi a-a y+\sin (y) \quad y \in[0,4 \pi]
$$

where $a>1$.

For both curves $\mu(y)=-a+\cos (y)$ and $v(y)=-\sin (y)$ and it follows that in both cases $\mathcal{F}(\Pi)$ is a circle with center $-a$ and radius 1 . In the case of $\Pi$ the circle is traversed once as $y$ increases, while in the case of $\Pi^{*}$ it is traversed twice. Knowledge of $\mathcal{F}(\Pi)$ alone is not enough to determine which curve generated it. The curves are not translations of each other. Assumption 5 rules this situation out. This example only considers a finite interval but it can be adapted so the functions are defined globally (see Appendix).

4 For a general curve $(x(t), y(t))$, not necessarily parameterized by arc length, the curvature is $\left(x^{\prime} y^{\prime \prime}-\right.$ $\left.y^{\prime} x^{\prime \prime}\right) /\left(x^{\prime 2}+y^{\prime 2}\right)^{3 / 2}$. 


\section{Conclusion}

This paper has consider the relationship between the elasticity and curvature of demand, as summarized in the demand manifold introduced by Mrázová and Neary (2017). It has shown that changes in demand leave the demand manifold, and so comparative statics, unchanged if and and only if they result from changes in market size and changes in quality.

Acknowledgements I am grateful to the Editor and an Associate Editor for helpful comments.

\section{Declarations}

Conflict of interest The author declares that he has no conflict of interest.

Open Access This article is licensed under a Creative Commons Attribution 4.0 International License, which permits use, sharing, adaptation, distribution and reproduction in any medium or format, as long as you give appropriate credit to the original author(s) and the source, provide a link to the Creative Commons licence, and indicate if changes were made. The images or other third party material in this article are included in the article's Creative Commons licence, unless indicated otherwise in a credit line to the material. If material is not included in the article's Creative Commons licence and your intended use is not permitted by statutory regulation or exceeds the permitted use, you will need to obtain permission directly from the copyright holder. To view a copy of this licence, visit http://creativecommons.org/licenses/by/4.0/.

\section{Appendix}

Equivalence of $\mathcal{D}(P)$ and $\mathcal{E}(P)$

Mrázová and Neary (2017) (Lemma 4 in Online Appendix B) note that

$$
\kappa=-\frac{1}{\epsilon}\left(1+\frac{1}{\epsilon}-\rho\right)
$$

$\eta=-1 / \epsilon$ so given $\epsilon$ and $\rho$ one can determine $\eta$ and $\kappa$ uniquely and vice-versa (and the mapping from one to another is smooth).

Details of Bipower case

Let

$$
P(x)=A x^{-\alpha}+B x^{-\beta}
$$

with $\alpha>\beta$. To be a valid demand function one requires $P(x) \geq 0$ for all $x$. For $x$ near zero $\operatorname{sgn} P(x)=\operatorname{sgn} A$, so $A>0$. One also requires $P^{\prime}(x) \leq 0$. For $x$ near zero $\operatorname{sgn} P^{\prime}=\operatorname{sgn}(-A \alpha)$ unless $\alpha=0$, in which case $\operatorname{sgn} P^{\prime}=\operatorname{sgn}(-B \beta)$. Hence either $\alpha>0$ or $\alpha=0$ and $B \beta>0$. If $B<0$ then $P\left(x_{\max }\right)=0$ for some finite $x_{\max }$. One can check that $P^{\prime}(x)<0$ for $0<x \leq x_{\max }$ under the assumptions made so far, so no further restrictions are needed. If $B>0$ then $\beta \geq 0$, as otherwise $P$ would be increasing for large $x$. 
Mrázová and Neary (2017) (Online Appendix B Proposition 3) show that $\mathcal{D}(P)$ has the form given in the text. The form of $\mathcal{E}(P)$ can be derived from this by using (19) or by direct calculation.

Direct calculation shows that

$$
\eta=-\frac{\alpha A x^{-\alpha}+\beta B x^{-\beta}}{A x^{-\alpha}+B x^{-\beta}}
$$

It is straightforward to check that as $x \rightarrow 0, \eta \rightarrow-\alpha$. If $B>0$ then, $x_{\max }=\infty$ and it is straightforward to check that $x \rightarrow-\beta$ as $x \rightarrow x_{\max }$ and $-\alpha<\eta(x)<-\beta$ for all $x$. If $B<0$, then $x_{\max }<\infty$ and it is again straightforward to check that $\eta(x) \rightarrow-\infty$ as $x \rightarrow x_{\max }$ and $-\infty<\eta(x)<-\alpha$ for all $x$.

To verify that Assumption 1 holds in the bipower case note that $\kappa$ only vanishes when $\eta=-\alpha$ or $\eta=-\beta$. From (21) it is easy to see that $\eta$ can only equal $-\alpha$ or $-\beta$ if $\alpha=\beta$.

Direct calculation shows that

$$
\kappa(x)=\frac{B A(\alpha-\beta)^{2} x^{\alpha-\beta}}{\left(A+B x^{\alpha-\beta}\right)^{2}}
$$

It follows that the sign of $\kappa$ is the same as that of $B$ for all $x$ as $A>0$. Hence if $B>0, \eta$ is monotone increasing and for $B<0, \eta$ is monotone decreasing. In either case, therefore, Assumptions 2 and 3 are satisfied.

Details of inverse exponential case

The expression for $\mathcal{D}(P)$ is derived by Mrázová and Neary (2017). The expression for $\mathcal{E}(P)$ can be derived from this using (19) or by direct calculation. The latter reveals that

$$
\kappa=\delta \eta-(\eta)^{2}-C \delta x^{\delta} \eta
$$

This can be used to eliminate $C x^{\delta}$ in terms of $\eta$ and $\kappa$ in the expression of $\eta$ as a function of $x$ :

$$
\eta=-\frac{B C x^{\delta} \exp \left(-C x^{\delta}\right)}{A+B \exp \left(-C x^{\delta}\right)}
$$

For Assumption 1, note that if $\kappa(x)=0$ then by differentiating (23) one obtains

$$
\frac{\partial^{3} \ln P}{(\partial \ln x)^{3}}=-C \delta^{2} \eta(x) \neq 0 \quad \text { when } \kappa(x)=0
$$

as $\eta(x)$ never vanishes, so Assumption 1 holds.

Assumption 2 follows as Mrázová and Neary (2017) note that

$$
\rho(x)=\delta C x^{\delta}-\delta+1
$$


(which is equivalent to (23)). This implies that the map $x \rightarrow \rho(x)$ is one-one. Since there is a one-one relation between $(\epsilon, \rho)$ and $(\eta, \kappa)$ this implies the assumption

For Assumption 3, note that if $A>0$ then $\eta \rightarrow 0$ as $x \rightarrow 0$ or $x \rightarrow \infty$ but $\eta(x)$ never vanishes for finite $x$, which implies the assumption. If $A<0$ then $p\left(x_{\max }\right)=0$ for some $x_{\max }$ and $\eta \rightarrow 0$ as $x \rightarrow 0$ and $\eta \rightarrow-\infty$ as $x \rightarrow x_{\max }$, which again implies the assumption.

Log-concavity implies subconvexity

By direct calculation

$$
\frac{d^{2} \ln P}{d(\ln x)^{2}}=x\left(\frac{d \ln P}{d x}+\frac{d^{2} \ln P}{d x^{2}}\right)
$$

So since $d \ln P / d x<0, d^{2} \ln P / d x^{2}<0$ implies $d^{2} \ln P / d(\ln x)^{2}<0$.

Proof of Proposition 2

Only the only if direction requires proof.

It follows from Assumption 4 and the inverse function theorem that $v(x)$ vanishes only for finitely many values of $y$ in any compact interval. Pick $y_{0}$ such that $v\left(y_{0}\right) \neq 0$. Consider the point $(\bar{\mu}, \bar{v})=\left(\mu\left(y_{0}\right), v\left(y_{0}\right)\right)$. Since $v\left(y_{0}\right) \neq 0$, by the inverse function theorem the map $y \rightarrow \mu(y)$ is locally invertible at $y_{0}$ and so locally $v$ can be written as a continuously differentiable function $h$ of $\mu$. Moreover Assumptions 5 and 6 imply that $g$ is a proper mapping (the inverse image of a compact set is compact), so Proposition 5.21 of Lee (2013) implies that $\mathcal{F}(\Pi)$ is an embedded submanifold of $\mathcal{R}^{2}$. This implies that the only branch of the demand manifold near $y_{0}$ is that described by $h$. More formally, it follows from these observations and Proposition 5.8 of Lee (2013) that one can find an open neighborhood $U$ of $(\bar{\mu}, \bar{v})$ and a continuously differentiable function $h$ so for $(\mu, v) \in U,(\mu, v) \in \mathcal{F}(\Pi)$ if and only if $v=h(\mu)$.

Since $\mathcal{F}(\Pi)=\mathcal{F}\left(\Pi^{*}\right)$, there exists $y_{0}^{*}$ such that $(\bar{\mu}, \bar{v})=\left(\mu^{*}\left(y_{0}^{*}\right), v^{*}\left(y_{0}^{*}\right)\right)$, where starred functions denote the corresponding functions of $\Pi^{*}$. One can choose intervals around $y_{0}$ and $y_{0}^{*}$ so that the images of $g$ and $g^{*}$ both lie in $U$. In these intervals $\Pi$ and $\Pi^{*}$ satisfy the second-order differential equations

$$
G^{\prime \prime}=h\left(G^{\prime}\right)
$$

as the images of $g$ and $g^{*}$ both lie on the curve defined by $h$.

Let $\beta=y_{0}-y_{0}^{*}$ and $\alpha=\Pi\left(y_{0}^{*}\right)-\Pi\left(y_{0}\right)$. Consider the function

$$
\bar{\Pi}(y)=\alpha+\Pi(y+\beta)
$$

By construction $\bar{\Pi}$ and $\Pi^{*}$ agree in value and first and second derivatives at $y_{0}^{*}$ (the latter being $\bar{\mu}$ and $\bar{v}$ ). Moreover $\bar{\Pi}$ and $\Pi^{*}$ both satisfy the differential equation (28). It follows by standard uniqueness theorems for solutions of differential equations (see for example the Lemma in Chapter 8.5 of Hirsch and Smale (1974)) that

$$
\Pi^{*}(y)=\bar{\Pi}(y)
$$

for a neighborhood around $y_{0}^{*}$. 
Let $\bar{y}$ be the supremum of the interval around $y_{0}^{*}$ for which (30) holds and let $y$ be the infimum. If $\bar{y}$ belongs to $\overline{\mathrm{dom}} \Pi^{*}$ and $\kappa(\bar{y}) \neq 0$ one can repeat the argument above and show that $\Pi^{*}(y)=\alpha^{\prime}+\Pi\left(y+\beta^{\prime}\right)$ for some $\alpha^{\prime}$ and $\beta^{\prime}$ in a neighborhood of $\bar{y}$. Since for $y<\bar{y}$ (30) holds, uniqueness for solutions of differential equations implies that $\alpha^{\prime}=\alpha$ and $\beta^{\prime}=\beta$. It follows that (30) holds for sufficiently small $y>\bar{y}$ as well, a contradiction to $\bar{y}$ being the supremum of values for which (30) holds. Similarly it cannot be the case that $\underline{y}$ belongs to $\overline{\operatorname{dom}} \Pi^{*}$ and $\kappa(\underline{y}) \neq 0$.

Suppose instead that $\kappa(\bar{y})=0$. Assumption 1 implies that $\kappa(y) \neq 0$ for $y \neq \bar{y}$ in a small neighborhood of $\bar{y}$. An analogous argument to that above shows that one can find $\alpha^{\prime}$ and $\beta^{\prime}$ so that $\Pi^{*}(y)=\alpha^{\prime}+\Pi\left(y+\beta^{\prime}\right)$ on the interval $(\bar{y}, \hat{y})$, for some $\hat{y}$. Since $\Pi$ and $\Pi^{*}$ are thrice continuously differentiable the first three derivatives of $\alpha+\Pi(y+\beta)$ and $\alpha^{\prime}+\Pi\left(y+\beta^{\prime}\right)$ must agree with those of $\Pi^{*}$ at $\bar{y}$. Since $g$ is one-one this implies that $\beta=\beta^{\prime}$ and hence $\alpha=\alpha^{\prime}$. This implies that (30) also holds on $[\bar{y}, \hat{y})$, a contradiction. This, and a similar argument for $y$ implies that (30) holds on all of $\overline{\operatorname{dom}} \Pi^{*}$, which yields the result.

Extension of Example 4

The example can be extended so that the functions are defined on the whole real line. Let

$$
k(y)=2 \pi a-a y-(y-2 \pi)^{2} \text { and } l(y)=2 \pi a+1-a y+1 /(y-1)^{3}
$$

and

$$
k^{*}(y)=4 \pi a-a y-(y-4 \pi)^{2} \text { and } l^{*}(y)=4 \pi a+1-a y+1 /(y-1)^{3}
$$

Finally let

$$
h(y)= \begin{cases}0 & y \leq 0 \\ \frac{e^{-1 / y}}{e^{-1 / y}+e^{-1 /(1-y)}} & 0<y<1 \\ 1 & y \geq 1\end{cases}
$$

$h$ is strictly increasing for $0<y<1$ and its derivatives of all orders vanish at $y=0$ and $y=1$.

Define

$$
\Psi(y)= \begin{cases}k(y) & y \leq-1 \\ (1-h(y+1)) k(y)+h(y+1) \Pi(y) & -1<y<0 \\ \Pi(y) & 0 \leq y \leq 2 \pi \\ (1-h(y-2 \pi)) \Pi(y)+h(y-2 \pi) l(y) & 2 \pi<y<2 \pi+1 \\ l(y) & y \geq 2 \pi+1\end{cases}
$$


and

$$
\Psi^{*}(y)= \begin{cases}k^{*}(y) & y \leq-1 \\ (1-h(y+1)) k^{*}(y)+h(y+1) \Pi^{*}(y) & -1<y<0 \\ \Pi^{*}(y) & 0 \leq y \leq 4 \pi \\ (1-h(y-4 \pi)) \Pi^{*}(y)+h(y-4 \pi) l^{*}(y) & 4 \pi<y<4 \pi+1 \\ l^{*}(y) & y \geq 4 \pi+1\end{cases}
$$

The graphs of $k$ and $k^{*}$ and of $l$ and $l^{*}$ are translations of each other. These are smoothly pasted onto those of $\Pi$ and $\Pi^{*}$ respectively to form $\Psi$ and $\Psi^{*}$. The latter are smooth and strictly decreasing functions. $\Psi$ and $\Psi^{*}$ satisfy Assumption 4.

The portions of the graph of $\Psi^{*}$ for $y \leq 0$ and for $y \geq 4 \pi$ are simply translations of those of $\Psi$ for $y \leq 0$ and $y \geq 2 \pi$ respectively. It follows that $\mathcal{F}(\Psi)=\mathcal{F}\left(\Psi^{*}\right)$ since $\mathcal{F}(\Pi)=\mathcal{F}\left(\Pi^{*}\right)$ but the overall graphs of $\Psi^{*}$ and $\Psi^{*}$ are not translations of one another since those of $\Pi$ and $\Pi^{*}$ are not.

\section{References}

Aguirre, I., Cowan, S., Vickers, J.: Monopoly price discrimination and demand curvature. Am. Econ. Rev. 100, 1601-1615 (2010)

Bulow, J., Pfleiderer, P.: A note on the effect of cost changes on prices. J. Polit. Econ. 91, 182-185 (1983) do Carmo, M.: Differential Geometry of Curves and Surfaces. Prentice-Hall, Englewood Cliffs, New Jersey (1976)

Hirsch, M., Smale, S.: Differential Equations, Dynamical Systems and Linear Algebra. Academic Press, Orlando, Florida (1974)

Kimball, M.: The quantitative analytics of the basic neomonetarist model. J. Money Credit Bank. 27, 1241-1277 (1995)

Lee, J.: Introduction to Smooth Manifolds, 2nd edn. Springer, New York (2013)

Mrázová, M., Neary, P.: Not so demanding: demand structure and firm behavior. Am. Econ. Rev. 107, 3835-74 (2017)

Mrázová, M., Neary, P.: Selection effects with heterogeneous firms. J. Eur. Econ. Assoc. 17, 1294-1334 (2019)

Weyl, G., Fabinger, M.: Pass-through as an economic tool. J. Polit. Econ. 123, 528-583 (2013)

Zhelobodko, E., Kokovin, S., Parenti, S., Thisse, J.F.: Monopolistic competition beyond the constant elasticity of subsitution. Econometrica 80, 2765-2784 (2012)

Publisher's Note Springer Nature remains neutral with regard to jurisdictional claims in published maps and institutional affiliations. 